\title{
Low Toxocara Seroprevalence in People in Rural Durango, Mexico
}

\author{
Cosme Alvarado-Esquivel ${ }^{1 *}$, Ángel Osvaldo Alvarado-Félix ${ }^{2}$ and Gustavo Alexis Alvarado-Félix ${ }^{2}$ \\ 'Biomedical Research Laboratory, Faculty of Medicine and Nutrition, Juárez University of Durango State, \\ Avenida Universidad S/N, 34000 Durango, Mexico \\ Colegio Anglo-Español Durango, Avenida Real del Mezquital 92, 34199, Durango, Mexico
}

Received: 27 Jun 2019; accepted: 10 Jul 2019

\begin{abstract}
The epidemiology of Toxocara infection in rural Mexico is largely unknown. Therefore, we sought to determine the seroprevalence of Toxocara infection in rural people in a northern Mexican state. We performed a cross-sectional seroprevalence study of 641 people living in rural Durango State including 282 subjects of the general population, 214 subjects of Huichol ethnicity, and 145 subjects of Mennonite ethnicity. Sera of participants were analyzed for the presence of anti-Toxocara immunoglobulin $\mathrm{G}(\mathrm{IgG})$ antibodies using a commercially available enzyme immunoassay. Three $(0.5 \%)$ of the 641 subjects tested were positive for anti-Toxocara IgG antibodies. Of the 3 Toxocara seropositive subjects, two were females, aged 19 and 39 years, and one was male, aged 59 years. They had contacted with dogs, cleaned cat excrement, consumed unwashed raw fruits, contacted soil, or lived in a house with soil floors. Seroprevalence of Toxocara infection was similar among the 3 groups of population studied: $0.4 \%$ for the general population, $0.9 \%$ for Huicholes, and $0.0 \%$ for Mennonites $(P=0.41)$. In conclusion, the Toxocara seroprevalence found in subjects in rural Durango is low as compared with those reported in people from rural areas in other countries.
\end{abstract}

Keywords: cross-sectional study, epidemiology, rural, ethnic groups, seroprevalence, toxocariasis

\section{Introduction}

The parasite Toxocara is the most ubiquitous intestinal nematode in dogs and cats [1]. This parasite is a zoonotic pathogen that causes toxocariasis and associated complications including allergic and neurological disorders [2]. Toxocariasis is one of the most commonly reported zoonotic helminth infections in the world [3]. Transmission to humans occurs by accidental infection of eggs present in, for instance, contaminated fruits or vegetables [4]. Toxocariasis is a neglected disease reaching high prevalence independently of the economic conditions [5]. Most human infections are asymptomatic [6]. Patients with toxocariasis may present fever, respiratory symptoms, gastrointestinal features [7], decreased visual acuity, eyestrain, headache, paleness [8], paresthesias, nervousness, and lipothymic states [9]. Ocular toxocariasis may lead to blindness [10]. Cardiac involvement is a rare but potentially life-threatening complication in Toxocara infection [11].

Living in rural areas is considered a risk factor for Toxocara infection as demonstrated in studies in several countries including Iran [12], Gabon [13], Korea [14, 15], Egypt [16], and Poland [17]. Knowledge about the seroepidemiology of Toxocara infection in rural Mexico is quite limited; however, we are aware of only one study about the seroepidemiology of Toxocara infection in rural Mexico. In a serosurvey of 126 Tepehuanos (an indigenous ethnic group) in rural Durango State, a $26.2 \%$ seroprevalence of Toxocara infection was found [18]. In the present study, we attempted to determine the seroprevalence of Toxocara infection and its association with the sociodemographic, clinical, and behavioral characteristics of the general population in the rural communities in the northern Mexican state of Durango. *Author for correspondence: Laboratorio de Investigación Biomédica, Facultad
de Medicina y Nutrición, Avenida Universidad S/N, 34000 Durango, Dgo, México; Tel/Fax: 0052-618-8130527; E-mail: alvaradocosme@yahoo.com

\section{Materials and Methods}

Study Design. We performed a cross-sectional serosurvey using serum samples from previous Toxoplasma gondii seroepidemiology studies [19-21]. The aims of the original studies were to determine the seroprevalence of and risk factors for Toxoplasma gondii infection in the studied population groups.

Study Population. We studied 641 people living in rural Durango State including 282 subjects of the general population, 214 subjects of Huichol ethnicity, and 145 subjects of Mennonite ethnicity. Inclusion criteria for enrollment were as follows: (1) living in rural Durango State, (2) aged 14 years and older, and (3) who accepted to participate in the survey. The socioeconomic status, gender, and educational level of the subjects were not restrictive criteria for enrollment. Concerning the 282 subjects of the general population, they were sampled in three communities: San Dimas, Villa Montemorelos, and Santa Clara, whereas Huicholes were sampled in the community of Huazamota. For its part, Mennonites were sampled in the community of Nuevo Ideal. In total, the tested subjects included 408 females and 233 males, aged 14-91 years (mean $40.30 \pm 16.69$ years).

Socio-Demographic, Clinical, and Behavioral Characteristics of the Subjects. We obtained the sociodemographic, clinical, and behavioral characteristics of the study population from archival Microsoft Excel files recorded in the original studies [19-21]. Sociodemographic data included age, gender, birthplace, occupation, socioeconomic status, educational level, and type of flooring at home. Clinical data included history of blood transfusion or solid organ transplantation. Behavioral data included animal contacts (cats, dogs, farm animals, and others), traveling (national or international trips), type of meat consumed (beef, pork, chicken, turkey, and others), degree of meat cooking (raw, undercooked, or well done), consumption of unpasteurized

This is an open-access article distributed under the terms of the Creative Commons Attribution-NonCommercial 4.0 International License (https://creativecommons.org/licenses/by-nc/4.0/), which permits unrestricted use, distribution, and reproduction in any medium for non-commercial purposes, provided the original author and source are credited, a link to the CC License is provided, and changes - if any - are indicated. 
milk, untreated water, unwashed raw fruits or vegetables, eating in restaurants or fast food outlets, and soil contact.

Detection of Anti-Toxocara IgG Antibodies. AntiToxocara immunoglobulin G (IgG) antibodies were detected in the sera of subjects using a commercially available enzyme immunoassay "Toxocara" kit (Diagnostic Automation, Inc. Calabasas, CA, USA). All assays were performed following the manufacturer's instructions. We included in each assay the negative and positive controls provided in the kit. Seropositivity was considered when an absorbance reading $\geq 0.3$ optical density units was obtained.

Statistical Analysis. We performed the statistical analysis using the software Microsoft Excel, Epi Info version 7, and SPSS version 20. We calculated the sample size using the following parameters: a population size of 500,000, a reference seroprevalence of $26.2 \%$ [18] as the expected frequency of exposure, $4 \%$ of confidence limits, and a $95 \%$ confidence level. The result of the sample size calculation was 464 subjects. We used the two-tailed Fisher's exact test to assess the association of Toxocara seropositivity and the sociodemographic, clinical, and behavioral characteristics of the subjects studied. A $P$ value $<0.05$ was considered statistically significant.

Ethical Aspects. In the present study, we analyzed only archival serum samples and data obtained in the previous studies. The original surveys were approved by Institutional Ethics Committees [19-21].

\section{Results}

Three $(0.5 \%)$ of the 641 subjects tested were positive for anti-Toxocara IgG antibodies. Of the 3 Toxocara seropositive subjects, 2 were females, aged 19 and 39 years, and one was male, aged 59 years. The occupations of these 3 seropositive subjects were as follows: a student, a housewife, and an agriculturist. They had contacted with dogs, cleaned cat excrement, consumed unwashed raw fruits, contacted soil, or lived in a house with soil floors. Seroprevalence of Toxocara infection was similar among the 3 groups of population studied: $0.4 \%$ for the general population, $0.9 \%$ for Huicholes, and $0.0 \%$ for Mennonites $(P=0.41)$. The Toxocara seropositivity rate did not vary $(P>0.05)$ with respect to sociodemographic characteristics of the study population including age, gender, birthplace, occupation, socioeconomic status, educational level, and type of flooring at home. Concerning clinical characteristics, none of the Toxocara seropositive individuals had a history of blood transfusion or solid organ transplantation. None of the behavioral characteristics analyzed including animal contacts, traveling, type of meat consumed, degree of meat cooking, consumption of unpasteurized milk, untreated water, unwashed raw fruits or vegetables, eating in restaurants or fast food outlets, and soil contact was associated with Toxocara seropositivity rate $(P>0.05)$.

\section{Discussion}

The seroepidemiology of Toxocara infection in rural Mexico has been sparsely studied so far. Therefore, in the current study, we sought to determine the seroprevalence of Toxocara infection in several communities in rural Durango State, Mexico. We found a low $(0.5 \%)$ seroprevalence of Toxocara infection in people living in rural areas of Durango State. This finding was unexpected since living in rural areas is considered as a risk factor for Toxocara exposure in several countries in Asia [12, 14, 15, 17], Africa [13, 16], and Europe [17]. The Toxocara seroprevalence found in our study is lower than those reported in rural populations in Brazil (71.8\%)
[22], Gabon (59.9\%) [13], Argentina (23\%-31.6\%) [23, 24], Poland (56.2\%) [17], Korea (5\%) [25], India (6.4\%) [26], Bolivia (34\%) [27], the Slovak Republic (17.09\%) [28], and Venezuela $(25.6 \%)$ [29]. In addition, the seroprevalence found in our study is lower than the $26.2 \%$ Toxocara seroprevalence reported in Tepehuanos in rural Durango, Mexico [18]. In fact, the seroprevalence found in our study is the lowest ever reported in rural communities. It is not clear why the seroprevalence in rural communities in Durango found in this study is lower than those reported in similar populations elsewhere. It is possible that the rate of Toxocara infection in dogs and cats and soil contamination with Toxocara in the rural communities explored was low. We cannot rule out previous deworming in cats and dogs to reduce parasite transmission in the communities studied. However, we did not obtain information about deworming in animals in the communities studied. We looked for socioeconomic, clinical, and behavioral factors associated with Toxocara infection in people in rural Durango; however, statistical analysis showed that none of the characteristics studied was associated with Toxocara infection. The three Toxocara seropositive individuals found in the current study had factors associated with Toxocara infection including contact with dogs, cleaning cat excrement, consumption of unwashed raw fruits, and soil contact. However, the lack of associations between Toxocara seroreactivity and the characteristics of the study population found in this study was probably due to the very low number of Toxocara seropositive individuals found. This low rate of Toxocara seropositivity was certainly a limitation of the survey. Additional studies with large sample sizes to determine risk factors associated with Toxocara exposure of people in rural Durango are needed.

In summary, we demonstrate a low rate of Toxocara exposure among people living in rural Durango State. The seroprevalence found is lower than those reported in people living in rural setting in other countries. Risk factors associated with Toxocara exposure in rural Mexico remain to be determined.

\section{Funding Sources}

This study was financially supported by Juárez University of Durango State.

\section{Authors' Contributions}

CAE designed the study protocol, performed the laboratory tests and data analysis, and wrote the manuscript. AOAF and GAAF performed the data analysis and reviewed the manuscript.

\section{Conflicts of Interest}

The authors declare no conflict of interest.

\section{References}

1. Chen J, Liu Q, Liu GH, Zheng WB, Hong SJ, Sugiyama H, et al. Toxocariasis: a silent threat with a progressive public health impact. Infect Dis Poverty. 2018;7(1):59. doi: 10.1186/s40249-018-0437-0.

2. Ma G, Holland CV, Wang T, Hofmann A, Fan CK, Maizels RM, et al. Human toxocariasis. Lancet Infect Dis. 2018;18(1):e14-e24. doi: 10.1016/ S1473-3099(17)30331-6.

3. Nicoletti A. Toxocariasis. Handb Clin Neurol. 2013;114:217-28. doi: 10.1016/B978-0-444-53490-3.00016-9.

4. Lötsch F, Vingerling R, Spijker R, Grobusch MP. Toxocariasis in humans in Africa - A systematic review. Travel Med Infect Dis. 2017;20:1525. doi: 10.1016/j.tmaid.2017.10.009.

5. Moreira GM, Telmo Pde L, Mendonça M, Moreira AN, McBride AJ, Scaini CJ, Conceição FR. Human toxocariasis: current advances in diagnostics, treatment, and interventions. Trends Parasitol. 2014;30(9):456-64. doi: 10.1016/j.pt.2014.07.003

6. Rubinsky-Elefant G, Hirata CE, Yamamoto JH, Ferreira MU. Human toxocariasis: diagnosis, worldwide seroprevalences and clinical expression of 
the systemic and ocular forms. Ann Trop Med Parasitol. 2010;104(1):3-23. doi: $10.1179 / 136485910 X 12607012373957$.

7. Ain Tiewsoh JB, Khurana S, Mewara A, Sehgal R, Singh A. Clinical and laboratory characteristics of patients with toxocariasis encountered at a tertiary care centre in North India. Indian J Med Microbiol. 2018;36(3):432434. doi: 10.4103/ijmm.IJMM 18259.

8. Martínez M, Montero J, Pineda A, Mijares V, Lares M, Catalano E, Ferrer E. Epidemiological, clinical and laboratory features of toxocariasis in school children from Aragua State, Venezuela. Trans R Soc Trop Med Hyg. 2018;112(6):255-263. doi: 10.1093/trstmh/try051.

9. Cojocariu IE, Bahnea R, Luca C, Leca D, Luca M. Clinical and biological features of adult toxocariasis. Rev Med Chir Soc Med Nat Iasi. 2012;116(4):1162-5

10. Martínez-Pulgarín DF, Muñoz-Urbano M, Gomez-Suta LD, Delgado OM, Rodriguez-Morales AJ. Ocular toxocariasis: new diagnostic and therapeutic perspectives. Recent Pat Antiinfect Drug Discov. 2015;10(1):35-41.

11. Kuenzli E, Neumayr A, Chaney M, Blum J. Toxocariasis-associated cardiac diseases-A systematic review of the literature. Acta Trop. 2016;154:107-20. doi: 10.1016/j.actatropica.2015.11.003.

12. Rezaiemanesh MR, Afzalaghaee M, Hamidi S, Eshaghzadeh A, Paydar M, Hejazi SH. Prevalence of toxocariasis and its related risk factors in humans, dogs and cats in northeastern Iran: a population-based study. Trans R Soc Trop Med Hyg. 2019. pii: trz011. doi: 10.1093/trstmh/trz011.

13. Lötsch F, Obermüller M, Mischlinger J, Mombo-Ngoma G, Groger M, Adegnika AA, et al. Seroprevalence of Toxocara spp. in a rural population in Central African Gabon. Parasitol Int. 2016;65(6 Pt A):632-634. doi: 10.1016/j. parint.2016.09.001.

14. Yang GL, Zhang XX, Shi CW, Yang WT, Jiang YL, Wei ZT, Wang CF, et al. Seroprevalence and associated risk factors of Toxocara infection in Korean, Manchu, Mongol, and Han ethnic groups in northern China. Epidemiol Infect. 2016;144(14):3101-3107.

15. Lee JY, Yang MH, Hwang JH, Kang M, Paeng JW, Yune S, Lee BJ, et al. The Prevalence of Toxocariasis and Diagnostic Value of Serologic Tests in Asymptomatic Korean Adults. Allergy Asthma Immunol Res. 2015;7(5):467-75. doi: 10.4168/aair.2015.7.5.467.

16. El-Shazly AM, Abdel Baset SM, Kamal A, Mohammed KA, Sakrs TI, Hammad SM. Seroprevalence of human toxocariasis (visceral larva migrans). J Egypt Soc Parasitol. 2009;39(3):731-44.

17. Zwoliński J. [The risk factors of Toxocara canis infestation in population of patients from the Lublin region]. Wiad Parazytol. 2000;46(4):463-73.
18. Alvarado-Esquivel C. Seroepidemiology of toxocariasis in a rural Tepehuanos population from Durango, Mexico. J Helminthol. 2014;88(2):1736. doi: $10.1017 / \mathrm{S} 0022149 \times 12000880$.

19. Alvarado-Esquivel C, Cruz-Magallanes HM, Esquivel-Cruz R, EstradaMartínez S, Rivas-González M, Liesenfeld O, et al. Seroepidemiology of Toxoplasma gondii infection in human adults from three rural communities in Durango State, Mexico. J Parasitol. 2008;94(4):811-6. doi: 10.1645/GE1524.1.

20. Alvarado-Esquivel C, Rojas-Rivera A, Estrada-Martínez S, SifuentesÁlvarez A, Liesenfeld O, García-López CR, Dubey JP. Seroepidemiology of Toxoplasma gondii infection in a Mennonite community in Durango State, Mexico. J Parasitol. 2010;96(5):941-5. doi: 10.1645/GE-2477.1.

21. Alvarado-Esquivel C, Pacheco-Vega SJ, Hernández-Tinoco J, SánchezAnguiano LF, Berumen-Segovia LO, Rodríguez-Acevedo FJ, Beristain-García I, et al. Seroprevalence of Toxoplasma gondii infection and associated risk factors in Huicholes in Mexico. Parasit Vectors. 2014;7:301. doi: 10.1186/ 1756-3305-7-301.

22. Araújo AC, Villela MM, Sena-Lopes Â, Farias NADR, Faria LMJ, Avila LFDC, et al. Seroprevalence of Toxoplasma gondii and Toxocara canis in a human rural population of Southern Rio Grande do Sul. Rev Inst Med Trop Sao Paulo. 2018;60:e28. doi: 10.1590/s1678-9946201860028.

23. Chiodo P, Basualdo J, Ciarmela L, Pezzani B, Apezteguía M, Minvielle M. Related factors to human toxocariasis in a rural community of Argentina. Mem Inst Oswaldo Cruz. 2006;101(4):397-400.

24. Fillaux J, Santillan G, Magnaval JF, Jensen O, Larrieu E, SobrinoBecaria CD. Epidemiology of toxocariasis in a steppe environment: the Patagonia study. Am J Trop Med Hyg. 2007;76(6):1144-7.

25. Park HY, Lee SU, Huh S, Kong Y, Magnaval JF. A seroepidemiological survey for toxocariasis in apparently healthy residents in Gangwon-do, Korea. Korean J Parasitol. 2002;40(3):113-7.

26. Malla N, Aggarwal AK, Mahajan RC. A serological study of human toxocariasis in north India. Natl Med J India. 2002;15(3):145-7.

27. Cancrini G, Bartoloni A, Zaffaroni E, Guglielmetti P, Gamboa H, Nicoletti A, Genchi C. Seroprevalence of Toxocara canis-IgG antibodies in two rural Bolivian communities. Parassitologia. 1998;40(4):473-5.

28. Havasiová K, Dubinský P, Stefancíková A. A seroepidemiological study of human Toxocara infection in the Slovak Republic. J Helminthol. 1993;67(4):291-6.

29. Lynch NR, Eddy K, Hodgen AN, Lopez RI, Turner KJ. Seroprevalence of Toxocara canis infection in tropical Venezuela. Trans R Soc Trop Med Hyg. 1988;82(2):275-81. 